S5 - Relative solvent accessibility (rSASA) values of natural substrates of proteases

Aspartate Protease

\begin{tabular}{|c|c|c|c|c|c|}
\hline Protein Name (PDB ID) & Protease Name & $\begin{array}{l}\text { Octapeptide } \\
\text { cleavage } \\
\text { sequence }\end{array}$ & rSASA & $\begin{array}{l}\text { Tetrapeptide } \\
\text { cleavage } \\
\text { sequence }\end{array}$ & rSASA \\
\hline \multirow[t]{3}{*}{$\begin{array}{ll}\text { ALPHA I-ANTICHYMOTRYPSIN } & \text { SERPIN } \\
(1 Q M N) & \\
\end{array}$} & \multirow[b]{3}{*}{ cathepsin D* } & LLSALVET & 0.82 & LSAL & 2.00 \\
\hline & & ITLLSALV & 2 & TLLS & 2.00 \\
\hline & & \begin{tabular}{|l} 
LSALVETR \\
\end{tabular} & 1 & SALV & 0.65 \\
\hline \multirow[t]{2}{*}{ PROLACTIN ANTAGONIST (2Q98) } & cathepsin D* & YPVWSGLP & 0.52 & PVWS & 0.34 \\
\hline & cathepsin $E^{* \pi}$ & NEIYPVWS & 0.74 & EIYP & 0.42 \\
\hline
\end{tabular}

\title{
Cysteine Protease
}

\begin{tabular}{|c|c|c|c|c|c|}
\hline Protein Name (PDB ID) & Protease Name & $\begin{array}{l}\text { Octapeptide } \\
\text { cleavage } \\
\text { sequence }\end{array}$ & rSASA & $\begin{array}{l}\text { Tetrapeptide } \\
\text { cleavage } \\
\text { sequence }\end{array}$ & rSASA \\
\hline \multirow[t]{3}{*}{ PROCATHEPSIN L (ICS8) } & \multirow[b]{3}{*}{ cathepsin $L^{* * *}$} & KVFQEPLF & 2 & VFQE & 0.46 \\
\hline & & LFYEAPRS & 2 & FYEA & 0.62 \\
\hline & & PLFYEAPR & 2 & LFYE & 0.68 \\
\hline SMALL G-PROTEIN (1MHI) & caspase-3 & DLRDDKDT & 0.75 & LRDD & 0.38 \\
\hline \multirow[t]{2}{*}{ ALPHA-1-ANTITRYPSIN (1QLP) } & \multirow[b]{2}{*}{ cathepsin $L^{* * *}$} & MFLEAIPM & 2 & FLEA & 0.66 \\
\hline & & AIPMSIPP & 2 & IPMS & 0.59 \\
\hline \multirow[t]{5}{*}{ HUMAN BCL-XL (IR2D) } & calpain-2 & WHLADSPA & 2 & HLAD & 2.00 \\
\hline & caspase-1 & HLADSPAV & 2 & LADS & 2.00 \\
\hline & \multirow[b]{2}{*}{ caspase-3 } & HLADSPAV & 2 & LADS & 2.00 \\
\hline & & SSLDAREV & 2 & SLDA & 2.00 \\
\hline & calpain-1 & EGTESEME & 2 & GTES & 2.00 \\
\hline BETA B2 CRYSTALLIN (1YTQ) & calpain-1 & HQTQAGKP & 2 & QTQA & 2.00 \\
\hline \multirow[t]{3}{*}{ EGFR KINASE DOMAIN (2ITV) } & calpain-1 & LWIPEGEK & 0.91 & WIPE & 0.31 \\
\hline & \multirow[b]{2}{*}{ caspase-3 } & DEEDMDDV & 1 & EEDM & 0.77 \\
\hline & & DMDDVVDA & 0.73 & MDDV & 0.49 \\
\hline P53DBD (2PCX) & caspase-3 & SDSDGLAP & 0.78 & DSDG & 0.65 \\
\hline \multirow[t]{2}{*}{ BETA-CATENIN (2Z6H) } & \multirow[b]{2}{*}{ caspase-3 } & YPVDGLPD & 2 & PVDG & 2.00 \\
\hline & & DLMDGLPP & 2 & LMDG & 2.00 \\
\hline \multirow[t]{2}{*}{ PROCATHEPSIN B (3PBH) } & \multirow[b]{2}{*}{ cathepsin $B^{* * * *}$} & YLKRLCGT & 2 & LKRL & 0.22 \\
\hline & & KRLCGTFL & 2 & RLCG & 0.14 \\
\hline
\end{tabular}

\section{Metallo Protease}

\begin{tabular}{|c|c|c|c|c|c|}
\hline Protein Name (PDB ID) & Protease Name & $\begin{array}{l}\text { Octapeptide } \\
\text { cleavage } \\
\text { sequence }\end{array}$ & rSASA & $\begin{array}{l}\text { Tetrapeptide } \\
\text { cleavage } \\
\text { sequence }\end{array}$ & ISASA \\
\hline STROMELYSIN (ICIZ) & $\begin{array}{l}\text { matrix metallopeptidase- } \\
13\end{array}$ & GIQSLYGP & 0.29 & IQSL & 0.23 \\
\hline APOLIPOPROTEIN E4 (1GS9) & $\begin{array}{l}\text { membrane-type matrix } \\
\text { metallopeptidase-1 }\end{array}$ & MDETMKEL & 0.42 & DETM & 0.24 \\
\hline \multirow[t]{3}{*}{ MMPO (GELATINASE B) (1L6J) } & matrix metallopeptidase-9 & TLKAMRTP & 0.65 & LKAM & 0.30 \\
\hline & \multirow[b]{2}{*}{ matrix metallopeptidase-3 } & DLGRFQTF & 0.63 & LGRF & 0.39 \\
\hline & & RVAEMRGE & 2 & VAEM & 2.00 \\
\hline
\end{tabular}




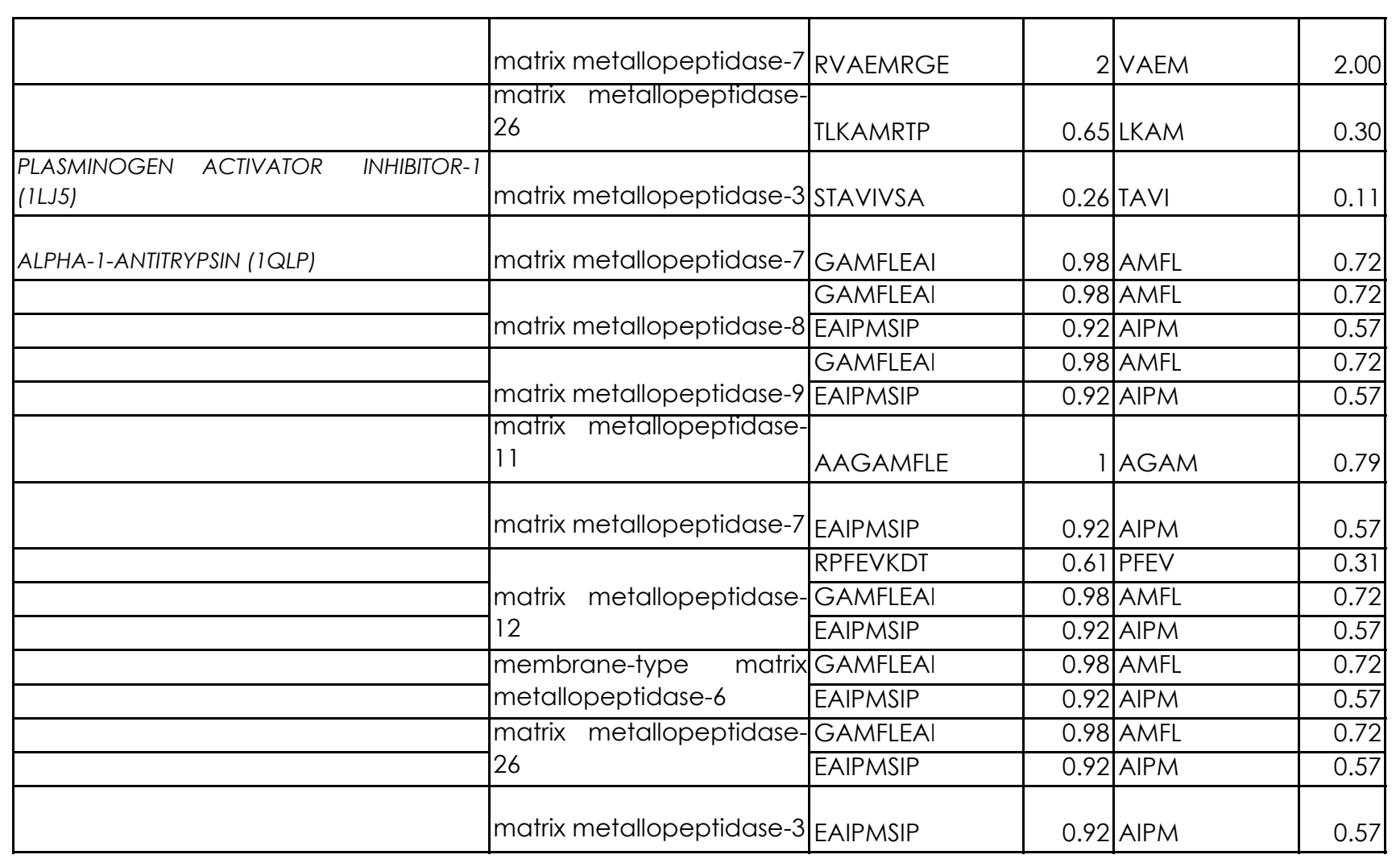

\section{Serine Protease}

\begin{tabular}{|c|c|c|c|c|c|}
\hline Protein Name (PDB ID) & Protease Name & $\begin{array}{l}\text { Octapeptide } \\
\text { cleavage } \\
\text { sequence }\end{array}$ & rSASA & $\begin{array}{l}\text { Tetrapeptide } \\
\text { cleavage } \\
\text { sequence }\end{array}$ & rSASA \\
\hline COMPLEMENT CIS PROTEASE (1ELV) & $\begin{array}{l}\text { complement component } \\
\text { activated } \mathrm{Clr}\end{array}$ & EKQRIIGG & 2 & $K Q R I$ & 2.00 \\
\hline GELATINASE A (IGEN) & elastase-2 & LGPVTPEI & 0.9 & GPVT & 0.88 \\
\hline \multirow[t]{2}{*}{ THROMBIN (1 JWT) } & coagulation factor $\mathrm{Xa}$ & IDGRIVEG & 1 & DGRI & 0.39 \\
\hline & thrombin & IDGRIVEG & 1 & DGRI & 0.39 \\
\hline \multirow[t]{2}{*}{\begin{tabular}{|ll} 
MMP9 & (GELATINASE B) (1L6J, \\
\end{tabular}} & chymase & FQTFEGDL & 0.64 & QTFE & 0.60 \\
\hline & trypsin-2 & DLGRFQTF & 0.63 & LGRF & 0.39 \\
\hline \multirow[t]{3}{*}{ HTRA protease (1LCY) } & \multirow[b]{3}{*}{ HtrA2 peptidase } & VRLLSGDT & 0.44 & RLLS & 0.34 \\
\hline & & PFALQNTI & 0.55 & FALQ & 0.30 \\
\hline & & LQNTITSG & 0.54 & QNTI & 0.39 \\
\hline $\begin{array}{lll}\text { PLASMINOGEN } & \text { ACTIVATOR } & \text { INHIBITOR-1 } \\
\text { (1LJ5) } & & \end{array}$ & matriptase-3 & VSARMAPE & 0.75 & SARM & 0.32 \\
\hline KALLIKREIN 6 (1LO6) & $\begin{array}{l}\text { kallikrein-related } \\
\text { peptidase } 6\end{array}$ & LRQRESSQ & 0.88 & RQRE & 0.63 \\
\hline COMPLEMENT PROTEASE CIR (IMD8) & $\begin{array}{l}\text { complement component } \\
\text { activated Clr }\end{array}$ & QRQRIIGG & 2 & RQRI & 2.00 \\
\hline SERUM ALBUMIN COMPLEXED (IN5U) & chymase & RETYGEMA & 0.53 & ETYG & 0.45 \\
\hline PRO-CHYMASE (1NN6) & chymase & PSQFNFVP & 0.68 & SQFN & 0.68 \\
\hline $\begin{array}{lll}\text { PLATELET RECEPTOR GLYCOPROTEIN IB- } \\
\text { ALPHA (IPOA) }\end{array}$ & cathepsin G & DTDLYDYY & 2 & TDLY & 2.00 \\
\hline FACTOR B (1QOP) & complement factor D & QQKRKIVL & 2 & QKRK & 2.00 \\
\hline ALPHA-1-ANTITRYPSIN (1QLP) & mesotrypsin & AAQKTDTS & 2 & AQKT & 2.00 \\
\hline
\end{tabular}


S5 - PDB hits with octapeptides

\begin{tabular}{|c|c|c|c|c|c|}
\hline ALPHA I-ANTICHYMOTRYPSIN SERPIN (IQMN) & elastase-2 & VKITLLSA & 2 & KITL & 2.00 \\
\hline CASPASE-3 (1QX3) & granzyme B & IETDSGVD & 2 & ETDS & 2.00 \\
\hline ABETA-BOUND HUMAN ABAD (1SO8) & HtrA2 peptidase & GNNCVFAP & 0.42 & NNCV & 0.32 \\
\hline VPS4B (IXWI) & HtrA2 peptidase & ILPIKFPH & 0.56 & LPIK & 0.30 \\
\hline FIBRILLARIN (2IPX) & granzyme B & VGPDGLVY & 0.39 & GPDG & 0.32 \\
\hline
\end{tabular}

*- Localized in Lysosome(primary), Extracellular,Golgi,ER

** - Localised in Endosome (primary), ER, Plasma Membrane, Cytoplast, Nucleus

*** - Localised in Endosome, Lysosome

**** - Localised in Lysosome (primary), Cytoplasm, Plasma Membrane, Mitochondria, Extracellular 